\title{
The particle gel immunoassay as a rapid test to rule out heparin-induced thrombocytopenia?
}

\author{
Katharina Schallmoser, MD, ${ }^{a}$ Camilla Drexler, MD, ${ }^{a}$ Eva Rohde, MD, ${ }^{a}$ Dirk Strunk, MD, ${ }^{b}$ Andrea Groselj-Strele, MSc, ${ }^{c}$ \\ Gerhard Lanzer, MD, ${ }^{\mathrm{a}}$ Hartmut Kroll, MD, ${ }^{\mathrm{d}}$ and Simon Panzer, MD, ${ }^{\mathrm{e}}$ Graz, Austria; Dessau, Germany; Vienna, Austria
}

Heparin-induced thrombocytopenia (HIT) is a prothrombotic condition characterized by a platelet decrease of $50 \%$ or thrombosis with a temporal relationship of 1 to 2 weeks after the initiation of heparin. These surrogate markers are useful for the clinical assessment ${ }^{1}$ but are hardly applicable in multimorbid patients with clinical conditions that mimic HIT. Platelet activation assays (heparin-induced platelet activation [HIPA] and serotonin release assay) and platelet factor 4 (PF4)/polyanion enzyme-linked immunosorbent assay (ELISA) confirm HIT. ${ }^{2}$ HIPA and serotonin release assay are highly specific, but they are laborious and require selected donor platelets and extended experience. High titer immunoglobulin ( $\mathrm{Ig}) \mathrm{G}$ antibodies correlate with clinical HIT, ${ }^{3}$ but ELISA is also time-consuming. The alternative heparin/PF4-antigen particle gel immunoassay (PaGIA) is easy, provides results within 1 hour, and detects mainly $\operatorname{IgG}$ but also $\operatorname{IgA} / \mathrm{M}$ antibodies. We evaluated the specificity and sensitivity of PaGIA in relation to HIPA and ELISA in 285 patients with an undetermined likelihood for HIT with the intention to validate it as a rapid assay to exclude HIT.

\section{PATIENTS AND METHODS}

Samples from 285 consecutive patients (median 71 years, range 1-97 years, $45 \%$ cardiovascular surgery; female/male $56 / 73$, and $55 \%$ medical, female/male 78/78) and 89 healthy controls (median 42 years, range 2664 years; female/male 49/40) were tested prospectively. The PaGIA (IDPaGIA H/PF4, DiaMed, Cressier s/Morat, Switzerland) was performed as described $^{4}$ and evaluated by 2 independent technicians. Briefly, heparin/ PF4-coated red particles become crosslinked in the presence of heparin antibodies and remain on top of a gel chamber after centrifugation. In the absence of antibodies, all particles sediment to the bottom of the gel chamber. A combined anti-human $\mathrm{IgG} / \mathrm{A} / \mathrm{M}$ and anti-human $\mathrm{IgG}$ only conjugate were used for the PF4-ELISA (PF4-ENHANCED; GTI, Waukesha, Wis) following the manufacturer's instructions. HIPA was performed as published. ${ }^{5}$ Analyses include chi-square, Mann-Whitney, receiver operating characteristic (ROC) curves (HIPA vs ELISA to deter-

\footnotetext{
From the University Clinic of Blood Group Serology and Transfusion Medicine, ${ }^{a}$ Department of Hematology and Stem Cell Transplantation, University Clinic of Internal Medicine, ${ }^{\mathrm{b}}$ Office for Biostatistics, Center for Medical Research, ${ }^{\mathrm{c}}$ Medical University of Graz, Austria; Institute for Transfusion Medicine Dessau, Red Cross Blood Transfusion Service NSTOB, ${ }^{\mathrm{d}}$ Dessau, Germany; and Clinical Department of Blood Group Serology, ${ }^{\mathrm{e}}$ Medical University of Vienna, Austria.

Received for publication Nov 27, 2007; accepted for publication March 18, 2008

Address for reprints: Simon Panzer, MD, Clinical Department of Blood Group Serology,

Medical University of Vienna, Waehringer Guertel 18-20, A-1090 Vienna, Austria

(E-mail: simon.panzer@meduniwien.ac.at).

J Thorac Cardiovasc Surg 2009;137:781-3

$0022-5223 / \$ 36.00$

Copyright $\subset 2009$ by The American Association for Thoracic Surgery

doi:10.1016/j.jtcvs.2008.03.044
}

mine their cutoff values), Bayes' theorem, and logistic regression. Data are presented as median and range.

\section{RESULTS}

HIPA was positive in $12 \%$ of patients. On the basis of ROC curves (ELISA vs HIPA), optical density (OD) cutoff values for the $\mathrm{IgG} / \mathrm{A} / \mathrm{M}$-ELISA and IgG-ELISA were 0.761 and 0.564 , respectively. In controls, the OD value of the IgG-ELISA was $0.066(0.028-0.500)$ and lower than from patients' samples, which were negative by $\mathrm{Pa}$ GIA and IgG/A/M-ELISA $(\mathrm{n}=158 ; 0.074 ; 0.009$ $0.339 ; P=.017)$. Of note, 2 male controls who had never received heparin had IgG-ELISA OD values of 0.425 and 0.500 . The latter serum was also positive by PaGIA, but both were HIPA negative. The OD values of the other 87 controls were less than 0.180 .

PaGIA was positive in 70 patients $(25 \%)$, and the relation to HIPA and ELISAs is shown in Table 1. In both patient groups, both ELISA ODs were higher if PaGIA and IgG/ A/M-ELISA were positive compared with negative PaGIA but positive $\operatorname{IgG} / \mathrm{A} / \mathrm{M}$-ELISA $(P<.001)$. The frequency of PaGIA-positive samples was similar among surgical and medical patients in all OD ranges (Figure 1). Three PaGIA-negative samples were HIPA positive, and one was IgG-ELISA positive.

On the basis of ROC curves (ELISA vs HIPA), IgG/A/ M-ELISA (OD cutoff 0.761) and IgG-ELISA (OD cutoff $0.564)$ had sensitivities of $81 \%$ and $86 \%$, and specificities of $75 \%$ and $81 \%$, respectively (positive and negative predictive values were $31 \%$ and $97 \%$ and $39 \%$ and $98 \%$, respectively, for IgG/A/M-ELISA and IgG-ELISA). Specificity, sensitivity, positive predictive value, and negative predictive value of PaGIA based on HIPA and ELISA are shown in Table 2. Logistic regression analysis showed that PaGIA, IgG/A/M-ELISA, and IgG-ELISA were significant predictors for the results of HIPA $(P<.001)$ and that the IgG-ELISA had the highest explained variance $(41.2 \%)$. There were no differences between male and female patients concerning specificity and sensitivity of any test.

\section{DISCUSSION}

The high mortality associated with HIT led to an increasing demand for its laboratory exclusion in patients with various clinical conditions that mimic HIT, particularly because multimorbid patients are more likely to form PF4 antibodies. ${ }^{2}$ The clinical score is highly reliable to exclude 
TABLE 1. Optical density values of the immunoglobulin-G/A/M enzyme-linked immunosorbent assay and immunoglobulin-G enzyme-linked immunosorbent assay in surgical and medical patients in relation to the results of the particle gel immunoassay

\begin{tabular}{|c|c|c|c|c|c|}
\hline & & & OD IgG/A/M ELISA & OD IgG ELISA & \\
\hline & PaGIA & IgG/A/M ELISA & Median (range) & Median (range) & \\
\hline \multirow[t]{4}{*}{ Surgical patients $n=129$} & Positive 28 & Positive 28 & $1.813(0.406-3.000)$ & $1.007(0.112-3.000)$ & \multirow{4}{*}{$P<.001$} \\
\hline & & Negative 0 & - & - & \\
\hline & Negative 101 & Positive 15 & $0.481(0.407-1.604)$ & $0.270(0.046-1.481)$ & \\
\hline & & Negative 86 & $0.154(0.060-0.395)$ & $0.068(0.021-0.339)$ & \\
\hline \multirow[t]{4}{*}{ Medical patients $n=156$} & Positive 42 & Positive 36 & $1.806(0.456-3.000)$ & $1.480(0.061-3.000)$ & \multirow{4}{*}{$P<.001$} \\
\hline & & Negative 6 & $0.157(0.114-0.339)$ & $0.117(0.044-0.233)$ & \\
\hline & Negative 114 & Positive 42 & $0.520(0.402-1.736)$ & $0.242(0.048-2.028)$ & \\
\hline & & Negative 72 & $0.181(0.050-0.397)$ & $0.076(0.020-0.313)$ & \\
\hline
\end{tabular}

PaGIA, Particle gel immunoassay; Ig, immunoglobulin; ELISA, enzyme-linked immunosorbent assay; OD, optical density.

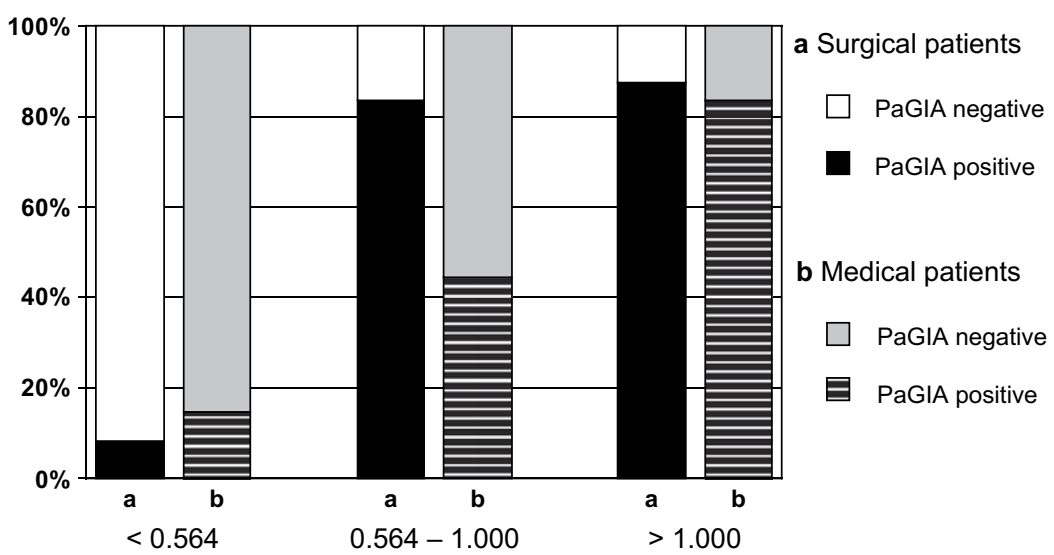

FIGURE 1. Distribution of surgical and medical patients with positive and negative PaGIA results in 3 OD intervals of the IgG-ELISA. The frequency of PaGIA-positive and negative samples was similar among surgical and medical patients in all OD ranges $(<0.564, P=.1 ; 0.564-1.000, P=.3$; $>1.000, P=1.0$ ). PaGIA, Particle gel immunoassay.

TABLE 2. Relation of the particle gel immunoassay to heparin-induced platelet activation test and to immunoglobulin-G/A/M and immunoglobulin-G enzyme-linked immunosorbent assay

\begin{tabular}{|c|c|c|c|c|c|c|}
\hline & \multicolumn{2}{|c|}{ PaGIA vs HIPA } & \multicolumn{2}{|c|}{ PaGIA vs IgG/A/M-ELISA } & \multicolumn{2}{|c|}{ PaGIA vs IgG-ELISA } \\
\hline & Surgical & Medical & Surgical & Medical & Surgical & Medical \\
\hline Sensitivity $\%$ & 71 & 79 & 92 & 73 & 86 & 73 \\
\hline Specificity $\%$ & 68 & 75 & 94 & 89 & 92 & 85 \\
\hline PPV \% & 21 & 32 & 79 & 69 & 67 & 57 \\
\hline NPV \% & 95 & 96 & 98 & 90 & 97 & 92 \\
\hline
\end{tabular}

PaGIA, Particle gel immunoassay; HIPA, heparin-induced platelet activation test; $I g$, immunoglobulin; ELISA, enzyme-linked immunosorbent assay; $P P V$, positive predictive value; $N P V$, negative predictive value.

HIT when clinical features are unambiguous, but a rapid assay is desirable for patients with undeterminable probability for HIT. Two commercialized assays provide results within 1 hour: the PaGIA and PIFA (Akers Biosciences, Thorofare, NJ). Reports to the Platelet Immunology Scientific Subcommittee (Geneva 2007) suggest a low specificity for the PIFA.

According to our results, the PaGIA can serve as a rapid test to exclude HIT because a negative PaGIA is rarely associated with high titer IgG PF4 antibodies or a positive HIPA.
Notably, PaGIA is negative in rare non-PF4 antibody-mediated HIT. ${ }^{5}$ Alternative anticoagulation should be considered until results from functional testing are available if the $\mathrm{Pa}-$ GIA is positive and in patients with a high probability for HIT and a negative PaGIA.

\section{References}

1. Lo GK, Juhl D, Warkentin TE, Sigouin CS, Eichler P, Greinacher A. Evaluation of pretest clinical score (4 T's) for the diagnosis of heparin-induced thrombocytopenia in two clinical settings. J Thromb Haemost 2006;4:759-65. 
2. Selleng K, Warkentin TE, Greinacher A. Heparin-induced thrombocytopenia in intensive care patients. Crit Care Med 2007;35:1165-76.

3. Zwicker JI, Uhl L, Huang WY, Shaz BH, Bauer KA. Thrombosis and ELISA optical density values in hospitalized patients with heparin-induced thrombocytopenia. J Thromb Haemost 2004;2:2133-7.

4. Pouplard C, Gueret P, Fouassier M, Ternisien C, Trossaert M, Régina S, et al. Prospective evaluation of the '4Ts' score and particle gel immunoassay specific to
heparin/PF4 for the diagnosis of heparin-induced thrombocytopenia. $J$ Thromb Haemost 2007;5:1373-9.

5. Eichler P, Budde U, Haas S, Kroll H, Loreth RM, Meyer O, et al. First workshop for detection of heparin-induced antibodies: validation of the heparin-induced plateletactivation test (HIPA) in comparison with a PF4/heparin ELISA. Thromb Haemost 1999;81:625-9.

\title{
A potential of autologous pericardium for a sustained-release carrier of vancomycin: A pilot study in vitro
}

\author{
Akira Marui, MD, PhD, ${ }^{\text {a }}$ Keiichi Hirose, MD, PhD, ${ }^{\text {a }}$ Hisashi Sakaguchi, MD, ${ }^{\text {a }}$ Yoshio Arai, MD, PhD, ${ }^{\text {a }}$ \\ Kazuhiko Doi, MD, PhD, ${ }^{\mathrm{b}}$ Masaki Tsukashita, MD, ${ }^{\mathrm{a}}$ Takeshi Shimamoto, MD, ${ }^{\mathrm{a}}$ Tadashi Ikeda, MD, PhD, ${ }^{\mathrm{a}}$ and \\ Masashi Komeda, MD, PhD, ${ }^{\text {a }}$ Kyoto, Japan
}

Autologous pericardium has been widely used to repair destructed annuli in infective endocarditis complicated by annular abscess. ${ }^{1,2}$ In the present study we investigated the in vitro property of autologous pericardium for a sustainedrelease carrier of vancomycin.

\section{CLINICAL SUMMARY}

Between January and May 2002, autologous pericardium with pericardial fat was harvested from the patients $(n=6)$ who underwent cardiac operations in our institute. After harvesting, the pericardium was cut into 7 pieces $(1 \mathrm{~cm} \times 1 \mathrm{~cm}$ each). Then $0.2 \mathrm{~mL}$ of vancomycin solution $(15 \mathrm{mg} / \mathrm{mL})$ was dropped onto each pericardium and incubated for 1 hour at room temperature so that the vancomycin solution was completely absorbed into the pericardium. The 7 pericardial patches with vancomycin were soaked in 7 test tubes containing $5 \mathrm{~mL}$ of saline, respectively. The test tubes were placed in the shaker and kept at $37^{\circ} \mathrm{C}$. At 6 hours, 12 hours, and $1,3,5,7$, and 10 days after the incubation, the pericardium and the saline samples were collected and frozen from 1 of the 7 test tubes, and the saline in the residual test tubes were replaced with $5 \mathrm{~mL}$ of fresh saline (ie, the pericardia were left uncollected), respectively. We replaced the saline in the residual test tubes at each time point to maintain the

\footnotetext{
From the Department of Cardiovascular Surgery, ${ }^{\text {a }}$ Kyoto University Graduate School of Medicine and the Department of Cardiovascular Surgery, ${ }^{\mathrm{b}}$ Takeda Hospital, Kyoto, Japan.

Received for publication Dec 9, 2007; revisions received Jan 18, 2008; accepted for publication Feb 4, 2008.

Address for reprints: Masashi Komeda, MD, PhD, Department of Cardiovascular Surgery, Kyoto University Graduate School of Medicine, 54 Shogoin-Kawahara, Sakyo, Kyoto, 606-8507 Japan (E-mail: komelab@kuhp.kyoto-u.ac.jp).

J Thorac Cardiovasc Surg 2009;137:783-4

$0022-5223 / \$ 36.00$

Copyright (C) 2009 by The American Association for Thoracic Surgery

doi:10.1016/j.jtcvs.2008.02.041
}

diffusion gradient between the pericardium and the saline. To prevent the degradation of the samples, we froze them until the concentrations of vancomycin were measured. The vancomycin concentrations of the pericardium and the saline samples were measured as previously described. ${ }^{3,4}$ We obtained written informed consent from each patient after a full explanation of this study. The protocol of this study complied with the principles set forth in the Helsinki Declaration.

All values are expressed as means \pm standard deviations. Figure 1 shows that the percentage reaming of vancomycin in the pericardium at each time point was $67.8 \% \pm 17.8 \%$, $53.8 \% \pm 12.3 \%, 37.8 \% \pm 9.8 \%, 25.1 \% \pm 10.1 \%, 12.6 \%$ $\pm 3.3 \%, 7.1 \% \pm 3.1 \%$, and $4.3 \% \pm 1.7 \%$ for 6 hours, 12 hours, and 1, 3, 5, 7, and 10 days after the incubation, respectively. Figure 2 shows that the concentrations of vancomycin in the saline samples were $789 \pm 143 \mu \mathrm{g} / \mathrm{mL}$, $376 \pm 56 \mu \mathrm{g} / \mathrm{mL}, 144 \pm 23 \mu \mathrm{g} / \mathrm{mL}, 56 \pm 14 \mu \mathrm{g} / \mathrm{mL}, 38 \pm$ $5.2 \mu \mathrm{g} / \mathrm{mL}, 27 \pm 3.8 \mu \mathrm{g} / \mathrm{mL}$, and $15 \pm 2.7 \mu \mathrm{g} / \mathrm{mL}$ for 6 hours, 12 hours, and 1, 3, 5, 7, and 10 days after the incubation, which were all greater than the minimum inhibitory concentration of vancomycin $(2.0 \mu \mathrm{g} / \mathrm{mL})$ against methicillin-resistant Staphylococcus aureus (MRSA). These results indicate that the pericardium can slowly release vancomycin and maintain the minimum inhibitory concentration of MRSA around the pericardium for more than 10 days.

\section{DISCUSSION}

In the present study we found that the autologous pericardium with fat might have a potential for a sustained-release carrier of vancomycin. Although this is an in vitro study and the mechanism of the sustained release was unclear, the property might help prevent prosthetic valve endocarditis by MRSA after reconstruction of the infected annulus.

Antibiotics are usually administrated systematically to prevent all forms of infection; however, this might be 